\title{
BILATERAL OPTIC ATROPHY IN CHILDHOOD. I
}

Bilateral optic atrophy is a common cause of blindness or defective vision in childhood and occurs in a number of disorders, some of which are genetically determined. When the presence of this defect is revealed ophthalmoscopically its various causes have to be borne in mind so that the conditions amenable to treatment are not missed and so that as accurate a prognosis as possible can be given.

In many instances it may be very difficult to differentiate between physiological and pathological pallor of the optic discs in young children, particularly when the two discs have a similar appearance. In some cases a lack of visual response may be thought to be due to optic atrophy when it is, in fact, the result of a congenital tapeto-retinal degeneration, of cortical blindness, or of a state of "apparent blindness" in which the absence of visual response is due to amentia. The accurate differentiation of these conditions requires the use of electro-diagnostic methods of investigation (Table).

TABLE

\begin{tabular}{l|c|c|c|c}
\hline Condition & $\begin{array}{c}\text { Optic } \\
\text { Atrophy }\end{array}$ & $\begin{array}{c}\text { Congenital } \\
\text { Tapeto-retinal } \\
\text { Degeneration }\end{array}$ & $\begin{array}{c}\text { Cortical } \\
\text { Blindness }\end{array}$ & $\begin{array}{c}\text { "Apparent” } \\
\text { Blindness }\end{array}$ \\
\hline Pupillary Light Reflexes & \pm & - & + & + \\
\hline Pendular Nystagmus & \pm & + & - & - \\
\hline Pallor of Optic Discs & + & \pm & - & - \\
\hline ERG & + & - & + & + \\
\hline VER & - & - & - & + \\
\hline
\end{tabular}

Atrophy of the optic nerves may be secondary to papilloedema or to a primary retinal disorder, but when there is no such precipitating local cause (primary optic atrophy) the cases may be broadly divided into three clinical groups: optic atrophy as an isolated finding; optic atrophy as the predominant clinical feature; and optic atrophy as only one aspect of a serious generalized disorder.

\section{(1) Hereditary Disorders}

\section{Optic Atrophy as an Isolated Finding}

There are three distinct hereditary forms of optic atrophy affecting children which differ from each other both in their modes of inheritance and in their clinical characteristics, this differentiation being most important in genetic councelling.

(a) Dominant Form.- Infantile optic atrophy, transmitted as an autosomal dominant trait, is present at birth or develops gradually in the first few years of life. The optic discs rarely become completely atrophic and in most cases central vision is only moderately impaired, varying from normal to $6 / 60$, and nystagmus is usually absent. Occasionally vision is severely impaired and in these uncommon cases nystagmus may be present. The peripheral visual fields are full and the electroretinogram (ERG) is normal. A defect of colour vision, usually a tritanopia, may occur.

(b) Recessive Form.-Congenital or infantile optic atrophy, transmitted as an autosomal recessive trait, is present at birth or occurs in early childhood, before the age of 3 years. The optic discs become completely atrophic and are often excavated, vision is severely affected and in some cases may fall to no perception of light, and nystagmus usually occurs. The peripheral visual fields are constricted but the ERG remains normal. Colour vision is usually severely affected.

(c) Leber's Optic Atrophy.-This form of optic atrophy, which is often preceded by an acute bilateral retrobulbar neuritis, usually develops after puberty (in males most commonly between the ages of 18 and 30 years, and in females between 10 and 30 years), although occasional cases occur in childhood. In the initial stages there may be slight oedema of the optic disc, followed by a 
variable degree of pallor. A relative central scotoma develops, more marked to coloured targets, and its presence is often indicated by an acquired defect of colour vision. In most cases the peripheral field of vision remains intact, but the central scotoma may break through to the temporal periphery.

Contrary to earlier views the disease is not inherited as an X-linked recessive trait as there is no evidence of the disorder ever having been transmitted by a male; the published pedigrees of Leber's optic atrophy cannot, indeed, be completely explained by any of the classical modes of mendelian inheritance.

There is some evidence that the basic abnormality in this disorder is an inherited biochemical defect in the mechanism for cyanide detoxication, so that increased cyanide exposure has a damaging effect on neuroretinal elements. This would explain the known association between Leber's optic atrophy and smoking, as the latter increases exogenous cyanide intake. In childhood a similarly increased cyanide intake may follow certain infections, particularly those involving the urinary and respiratory tracts, and such infections closely precede the onset of optic neuropathy in some cases. The use of high doses of hydroxycobalomin has been advocated during the early stages of the disorder because of its known antagonism to cyanide, and some success has been claimed from such treatment in both children and adults.

\section{(2) Non-hereditary Disorders}

When there is no family history of a similar disorder, bilateral optic atrophy in a child may in some cases be the result of a new mutation or to the first appearance of an autosomal recessive trait, but in others it is an acquired disorder, the commonest and most important causes of which are the following:

(a) Glioma of the Optic Chiasma and Craniopharyngioma.-When bilateral optic atrophy is found in a child it is always most important to exclude an acquired cause, of which a tumour is the most important, before assuming that the atrophy is developmental or congenital in origin. A glioma of the optic chiasma causes progressive bilateral optic atrophy, often without any other symptoms or signs, although see-saw or rotatory nystagmus and an insidious increase in the circumference of the skull may be present in some cases. Similarly, a craniopharyngioma may cause optic atrophy in an otherwise apparently normal child, although its presence may be determined radiographically by the characteristic rim of calcification corresponding to the cyst. Radiographic examination of the sella turcica and of the optic foramina should therefore be carried out in all children exhibiting optic atrophy of uncertain aetiology in order to exclude these two tumours. In addition, glioma is more common in children with neurofibromatosis and careful examination of the skin may reveal areas of café-au-lait pigmentation even in the absence of other clinical manifestations of this phakomatosis.

(b) Demyelinating Optic Neuritis.-In childhood, isolated optic neuritis usually occurs as a localized form of encephalomyelitis of uncertain aetiology rather than as a sign of multiple sclerosis. After the acute phase of the neuropathy there may be a permanent residual visual defect with bilateral pallor of the optic discs.

(c) Drug Toxicity.-The most important drugs which may cause a toxic optic neuritis and optic atrophy in children are streptomycin and isoniazid, the sulphonamides and chloramphenicol. The first two may be prescribed over a long period in the treatment of tuberculosis, and the last in the long-term treatment of the recurrent pulmonary infections which occur in children suffering from fibrocystic disease (mucoviscidosis).

(d) Malnutrition.-A condition of tropical amblyopia has been described wherein bilateral optic atrophy, usually arising in childhood, is found as an isolated pathological process. The condition is most commonly seen in West Indian Negroes and, although its exact aetiology is not fully understood, it is thought to be the result of malnutrition associated with avitaminosis, the effects of which are possibly made worse by the additional toxic influence of the cyanides present in "cassava" or "bush tea".

(To be concluded)

Brian Harcourt AND Barrie Jay, INSTITUTE OF OPHTHALMOLOGY, LONDON, W.C.1. 